\title{
Identification and clinical significance of an elevated level of serum aminoacylase-1 autoantibody in patients with hepatitis B virus-related liver cirrhosis
}

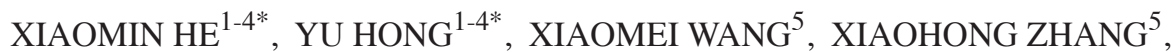 \\ JIANG LONG ${ }^{6}$, HAI LI ${ }^{7}$, BEI ZHANG ${ }^{1-4}$, SUHONG CHEN $^{8}$, QIQI LIU ${ }^{8}$, HONGYI LI $^{1-4}$, \\ XIAOMING WANG ${ }^{1-4}$, XIAOJUAN OU ${ }^{1-4}$ and JIAN HUANG ${ }^{1-4}$ \\ ${ }^{1}$ Liver Research Center, Beijing Friendship Hospital, Capital Medical University; \\ ${ }^{2}$ Beijing Key Laboratory of Translational Medicine in Liver Cirrhosis; \\ ${ }^{3}$ Beijing Key Laboratory of Tolerance Induction and Organ Protection in Transplantation; \\ ${ }^{4}$ National Clinical Research Center for Digestive Diseases, Beijing 100050; \\ ${ }^{5}$ Department of Obstetrics, Beijing Obstetrics and Gynecology Hospital, Capital Medical University, Beijing 100026; \\ ${ }^{6}$ Department of Oncology Minimally Invasive Interventional Radiology, Beijing You-an Hospital, Capital Medical University, \\ Beijing 100069; ${ }^{7}$ Department of Hepatopancreatobiliary and Splenic Medicine, \\ Affiliated Hospital of Medical College of Chinese People's Armed Police Force, Tianjin 300192; \\ ${ }^{8}$ Biotechnology Center, Beijing Institute of Radiation Medicine, Beijing 100850, P.R. China
}

Received August 2, 2015; Accepted August 10, 2016

DOI: $10.3892 / \mathrm{mmr} .2016 .5740$

\begin{abstract}
Chronic hepatitis B (CHB) is prevalent worldwide and can develop into liver cirrhosis and liver carcinoma. Early discrimination of liver cirrhosis from chronic hepatitis is critical for effective treatment and optimal prognosis. The aim of the present study was to assess the diagnostic value of
\end{abstract}

Correspondence to: Professor Jian Huang or Dr Xiaojuan Ou, Liver Research Center, Beijing Friendship Hospital, Capital Medical University, 95 Yong An Road, Beijing 100050, P.R. China

E-mail: huangj1966@hotmail.com

E-mail: ouxj16@sina.com

*Contributed equally

Abbreviations: ACY1, aminoacylase-1; IMP-2, insulin-like growth factor 2 mRNA-binding protein 2; CRT, calreticulin; CENPF, centromere protein F; HSP60, $60 \mathrm{kDa}$ heat shock protein; PDIA1, protein disulfide-isomerase; ENO1, $\alpha$-enolase; ANXA4, annexin $\mathrm{A} 4$; IGKC, Ig $\kappa$ chain $\mathrm{C}$ region; RGN, regucalcin; $\mathrm{K} 2 \mathrm{C} 1$, type II cytoskeletal 1; HSPA6, heat shock $70 \mathrm{kDa}$ protein 6; AIAT, $\alpha$-1-antitrypsin; FGB, fibrinogen $\beta$ chain; SBP1, selenium-binding protein 1; PRDX3, peroxiredoxin 3; HINT1, histidine triad nucleotide-binding protein 1 ; TUBB4B, tubulin $\beta$-4B chain; ATPB, ATP synthase subunit $\beta$; ALDH1A1, retinal dehydrogenase 1; hnRNP A2, heterogeneous nuclear ribonucleoprotein A2; AIF, apoptosis-inducing factor

Key words: autoantibody, biomarker, chronic hepatitis $\mathrm{B}$, HBV-related liver cirrhosis a panel of cellular proteins that can be recognized by autoantibodies in patient serum for hepatitis B virus (HBV)-related liver cirrhosis. Twenty-two candidate autoantigens screened using a serum proteomics assay in our previous study were assessed retrospectively in 443 participants, comprising 89 patients with HBV-related liver cirrhosis, 89 patients with $\mathrm{CHB}$, and 265 healthy controls. The levels of autoantibodies against the candidate autoantigens were measured by protein microarrays containing the candidate antigen proteins. Receiver operating characteristic (ROC) curves were used to calculate the diagnostic accuracy. The present study determined that seven of the 22 candidate autoantibodies differed significantly in serum level between HBV-related liver cirrhosis and CHB $(\mathrm{P}<0.0001)$, with area under curve (AUC) values $>0.7$. The seven autoantibodies recognized aminoacylase-1 (ACY1), histidine triad nucleotide-binding protein 1, insulin-like growth factor 2 mRNA-binding protein 2 , heat shock $70 \mathrm{kDa}$ protein 6 , peroxiredoxin 3, apoptosis-inducing factor and regucalcin. Among these, the ACY1 autoantibody had the highest value for discriminating HBV-related liver cirrhosis from $\mathrm{CHB}$, with an AUC value of 0.872 (95\% confidence interval: $0.810-0.934, \mathrm{P}<0.0001$ ), sensitivity of $77.3 \%$ and specificity of $85.0 \%$. In conclusion, with the elevated level in the disease progression of CHB, ACY1 autoantibody may be a valuable serum biomarker for discriminating HBV-related liver cirrhosis from CHB.

\section{Introduction}

Hepatitis B virus (HBV) infection is an important cause of chronic hepatitis and remains a significant public health issue worldwide. An estimated 350 million individuals are chronically infected with HBV worldwide $(1,2)$. Patients with 
chronic hepatitis have a high risk of progression to cirrhosis and hepatocellular carcinoma (HCC), and early diagnosis of liver cirrhosis is essential for their prognosis. The diagnosis of liver cirrhosis is usually achieved by liver biopsy, imaging examinations, and clinical signs. However, use of a serum biomarker for diagnosis of liver cirrhosis is rare.

Autoantibodies comprise a novel type of serum biomarker identified in recent years. Based on the various environments in diseases, abnormal proteins may lead to antigenicity, which can drive the humoral immune responses to produce autoantibodies. The presence of serum autoantibodies has been observed not only in autoimmune diseases, but also in non-autoimmune diseases, such as cancer (3-5). A recent study showed that autoantibodies against $\alpha$-enolase (ENO1) had potential diagnostic value in liver fibrosis (6). Furthermore, autoantibodies may have various advantages as immunodiagnostic markers, as the magnified signals of autoantibodies can be easier to detect than the autoantigens themselves $(3,7)$.

Our previous study, identified potential tumor-associated antigens in hepatocellular carcinoma using serum proteomics analysis (8). Notably, there were elevated levels of autoantibodies in raised against some of these autoantigens in patients with liver cirrhosis. Chronic hepatitis patients have a high risk of progression to cirrhosis and $\mathrm{HCC}$, and early diagnosis of liver cirrhosis is essential for their prognosis. Therefore, in the present study, our established protein chip technology (8) was used to evaluate the diagnostic value of these autoantibodies in liver cirrhosis with the aim of distinguishing HBV-related liver cirrhosis from chronic hepatitis B (CHB), and to provide the basis for further research on autoantibody biomarkers for staging liver fibrosis.

\section{Materials and methods}

Patients. A total of 443 participants were recruited for clinical evaluation, comprising 89 patients with HBV-related liver cirrhosis (65 men and 24 women, aged 27-89 years with a median age of 46.0 years), 89 patients with CHB (61 men and 28 women, aged 23-75 years with a median age of 39.3 years), and 265 healthy controls (166 men and 99 women, aged 20-66 years with a median age of 45.8 years). The patients were retrospectively recruited between 2010 and 2014 from the Liver Research Center, Beijing Friendship Hospital, Capital Medical University, Department of Minimally Invasive Interventional Radiology, Beijing Youan Hospital, Capital Medical University, and Department of Hepatopancreatobiliary and Splenic Medicine, Affiliated Hospital of Medical College of Chinese People's Armed Police Force. The healthy controls were qualified blood donors with normal liver biochemistry, no history of liver disease and no malignant disease.

Inclusion criteria: The diagnosis of CHB included the presence of HBsAg $\geq 6$ months, HBV DNA concentrations $\geq 10^{3}$ copies $/ \mathrm{ml}$, and elevation of serum alanine aminotransferase (9), without evidence of hepatitis C virus infection or a history of alcohol abuse. The diagnosis of liver cirrhosis met at least one of the following three criteria: i) Histologically confirmed cirrhosis (Ishak 5/6 or Metavir F4); ii) Presence of portal hypertension (endoscopy showing esophageal varices, or imaging showing liver surface nodularity, splenomegalia or hypersplenism) and liver dysfunction (albumin $<35.0 \mathrm{~g} / \mathrm{l}$, or International Normalized Ratio $>1.3$ ); iii) chronic liver disease patients experiencing variceal bleeding, ascites or encephalopathy. Patients with autoimmune liver diseases, such as autoimmune hepatitis, primary biliary cirrhosis, and other autoimmune diseases, such as systemic lupus erythematosus, rheumatoid arthritis, and diabetes mellitus, were excluded from the study.

All serum samples were stored at $-80^{\circ} \mathrm{C}$ until testing. The study protocol was approved by the Clinical Research Ethics Committee of Beijing Friendship Hospital, Capital Medical University (Beijing, China).

Protein microarray analysis for clinical evaluation of candidate autoantigens. Recombinant proteins for candidate autoantigens screened in our previous study available for enzyme-linked immunosorbent assay were purchased when required, including including insulin-like growth factor 2 mRNA-binding protein 2 (IMP-2), calreticulin (CRT), centromere protein F (CENPF), $60 \mathrm{kDa}$ heat shock protein (HSP60), protein disulfide-isomerase (PDIA1), aminoacylase-1 (ACY1), $\alpha$-enolase (ENO1), annexin A4 (ANXA4), Ig $\kappa$ chain $\mathrm{C}$ region (IGKC), Regucalcin (RGN), type II cytoskeletal $1(\mathrm{~K} 2 \mathrm{C} 1)$, heat shock $70 \mathrm{kDa}$ protein 6 (HSP A6), $\alpha$-1-antitrypsin (AIAT), fibrinogen $\beta$ chain (FGB), selenium-binding protein 1 (SBP1), peroxiredoxin 3 (PRDX3), histidine triad nucleotide-binding protein 1 (HINT1), tubulin $\beta-4 B$ chain (TUBB4B), ATP synthase subunit $\beta$ (ATPB) and retinal dehydrogenase 1 (ALDH1A1). Two other autoantigens, heterogeneous nuclear ribonucleoprotein A2 (hnRNP A2) and apoptosis-inducing factor (AIF), were prepared as described previously (8).

Preparation and detection of the protein microarray were performed according to our previous study (8). Briefly, the screened protein antigens were diluted to individually optimized concentrations and robotically attached to aldehyde-activated glass slides in ordered arrays by a computer-controlled microchip spotting instrument (Cartesian Technologies, Irvine, CA, USA). Human IgG (Sigma-Aldrich; Merck Millipore, Darmstadt, Germany) was used as a positive control and an internal standard for signal intensity calibration in each test, while sample liquid and bovine serum albumin (Sigma-Aldrich; Merck Millipore) were used as negative controls.

The prepared antigen microarrays were blocked in PBS containing 25\% fetal bovine serum (Sigma-Aldrich; Merck Millipore) at $37^{\circ} \mathrm{C}$ for $2 \mathrm{~h}$ and then washed with PBST [0.01 mol/1 PBS (pH 7.2-7.4) with 0.05\% Tween 20] for $10 \mathrm{sec}$ and repeated three times. Then the serum samples were added to the microarray at 1:5 dilution with PBS with a $10 \mu 1$ sample for each matrix and incubated at $37^{\circ} \mathrm{C}$ for $30 \mathrm{~min}$. The microarray was washed six times with PBST and incubated with horseradish peroxidase-labeled rabbit anti-human $\operatorname{IgG}$ (cat. no. A8792; Sigma-Aldrich; Merck Millipore) with a working concentration of $1: 8,000$ for a further $30 \mathrm{~min}$ at $37^{\circ} \mathrm{C}$ and followed by six repetitions of PBST washing. After it was dried at room temperature, the immunoreactive spots were detected by an enhanced chemiluminescence Western Blotting kit (Merck Millipore) using a PLNT YQ001 scanner (Puli 
Table I. Diagnostic value of autoantibodies to distinguish hepatitis B virus-related liver cirrhosis from chronic hepatitis B.

\begin{tabular}{|c|c|c|c|c|c|c|c|c|}
\hline Autoantibody & $\begin{array}{l}\text { AUC } \\
\text { value }\end{array}$ & $95 \% \mathrm{CI}$ & $\begin{array}{l}\text { Cut-off } \\
\text { value }\end{array}$ & $\begin{array}{c}\text { Sensitivity } \\
(\%)\end{array}$ & $\begin{array}{c}\text { Specificity } \\
(\%)\end{array}$ & $\begin{array}{l}\text { PPV } \\
(\%)\end{array}$ & $\begin{array}{c}\text { NPV } \\
(\%)\end{array}$ & P-value \\
\hline ACY1 & 0.872 & $0.810-0.934$ & 1920 & 77.3 & 85.0 & 85.0 & 77.3 & $<0.0001$ \\
\hline HINT1 & 0.794 & $0.709-0.878$ & 1856 & 85.5 & 70.0 & 72.3 & 84.0 & $<0.0001$ \\
\hline IMP-2 & 0.777 & $0.691-0.863$ & 896 & 62.3 & 92.9 & 88.4 & 73.9 & $<0.0001$ \\
\hline PRDX3 & 0.777 & $0.696-0.859$ & 1728 & 54.5 & 90.0 & 85.7 & 64.3 & $<0.0001$ \\
\hline HSP A6 & 0.765 & $0.682-0.848$ & 1600 & 58.5 & 83.3 & 79.2 & 64.9 & $<0.0001$ \\
\hline AIF & 0.756 & $0.675-0.837$ & 5504 & 60.0 & 80.0 & 80.0 & 60.0 & $<0.0001$ \\
\hline RGN & 0.711 & 0.617-0.804 & 640 & 86.1 & 53.3 & 68.9 & 76.2 & $<0.0001$ \\
\hline hnRNP A2 & 0.669 & $0.583-0.755$ & 1664 & 61.4 & 66.1 & 72.0 & 54.7 & $<0.0001$ \\
\hline ATPB & 0.666 & $0.595-0.738$ & 704 & 68.2 & 59.6 & 68.8 & 59.0 & $<0.0001$ \\
\hline HSP60 & 0.665 & $0.580-0.750$ & 4672 & 59.7 & 68.2 & 54.1 & 59.0 & $<0.0001$ \\
\hline SBP1 & 0.664 & $0.571-0.756$ & 704 & 74.0 & 56.7 & 67.5 & 64.2 & 0.001 \\
\hline FGB & 0.657 & $0.564-0.751$ & 1088 & 65.0 & 65.0 & 71.2 & 58.2 & 0.001 \\
\hline IGKC & 0.654 & $0.556-0.751$ & 2112 & 76.9 & 60.6 & 58.8 & 78.2 & 0.004 \\
\hline CRT & 0.633 & $0.534-0.731$ & 704 & 44.4 & 83.3 & 73.7 & 58.8 & 0.011 \\
\hline CENPF & 0.630 & $0.540-0.721$ & 1088 & 69.0 & 61.0 & 62.0 & 68.1 & 0.006 \\
\hline PDIA1 & 0.618 & $0.524-0.712$ & 704 & 54.3 & 72.7 & 64.4 & 63.6 & 0.013 \\
\hline ANXA4 & 0.618 & $0.524-0.712$ & 448 & 73.8 & 48.3 & 65.6 & 64.4 & 0.017 \\
\hline ENO1 & 0.595 & $0.510-0.679$ & 896 & 44.8 & 72.1 & 61.9 & 56.4 & 0.032 \\
\hline
\end{tabular}

ACY1, aminoacylase-1; HINT1, histidine triad nucleotide-binding protein 1; IMP-2, insulin-like growth factor 2 mRNA-binding protein 2; PRDX3, peroxiredoxin 3; HSP A6, heat shock $70 \mathrm{kDa}$ protein 6; AIF, apoptosis-inducing factor; RGN, regucalcin; hnRNP A2, heterogeneous nuclear ribonucleoprotein A2; ATPB, ATP synthase subunit $\beta$; HSP60, 60 kDa heat shock protein; SBP1, selenium-binding protein 1; FGB, fibrinogen $\beta$ chain; IGKC, Ig $\kappa$ chain C region; CRT, calreticulin; CENPF, centromere protein F; PDIA1, protein disulfide-isomerase; ANXA4, annexin A4; ENO1, $\alpha$-enolase; AUC, area under curve; 95\% CI, 95\% confidence interval; PPV, positive predictive value; NPV, negative predictive value.

Knight Biotechnology Co. Ltd, Beijing, China). The signal intensities of the spots and the background values were measured by Array Vision 7.0 (Imaging Research, St. Catharines, Canada).

Statistical analysis. Statistical analyses were performed with SPSS version 19.0 (SPSS Inc., Chicago, IL, USA) and MedCalc version 10.4.7.0 (MedCalc Software, Oostende, Belgium). Receiver-operating characteristic (ROC) curves were used to assess the sensitivity, specificity, and area under curve (AUC) value with $95 \%$ confidence interval $(95 \% \mathrm{CI})$ to evaluate the diagnostic value of the serum markers. The optimum cut-off values were determined by calculating the Youden index, and the corresponding signal intensity number was set as the cut-off value for positivity of individual autoantibodies to protein antigens. The correlation between clinical parameters and autoantibody positivities were analyzed by the $\chi^{2}$ test with Yate's correction. $\mathrm{P}<0.05$ was considered to indicate a statistically significant difference.

\section{Results}

High-throughput clinical evaluation. For the high-throughput clinical evaluation, 22 candidate protein antigens were used for protein microarrays: IMP-2, CRT, CENPF, HSP60, PDIA1, ACY1, ENO1, ANXA4, IGKC, RGN, K2C1, HSP A6, AIAT, FGB, SBP1, PRDX3, HINT1, TUBB4B, ATPB, ALDH1A1,
AIF and hnRNP A2. A schematic representation of the antigen array is shown in Fig. $1 \mathrm{~A}$ and $\mathrm{B}$, and representative scanned images of protein microarrays are shown in Fig. 1C.

After microarray detection with the 443 serum samples, ROC curves were generated for all 22 protein antigens based on the individual signal intensities. The results showed that the autoantibodies against ACY1, HINT1, IMP-2, PRDX3, HSP A6, AIF and RGN differed significantly $(\mathrm{P}<0.05)$ between HBV-related liver cirrhosis and CHB patients, with an AUC value of $>0.7$, which is a recognized standard for biomarkers that have a promising diagnostic value in general (Fig. 2A, Table I). The levels of autoantibodies against these protein antigens in $\mathrm{CHB}$ and $\mathrm{HBV}$-related liver cirrhosis showed trends toward increases. Among them, the ACY1 autoantibody showed the highest diagnostic value for HBV-related liver cirrhosis, with AUC value of 0.872 , a sensitivity of $77.3 \%$ and specificity of $85.0 \%$ (Table I).

Mean signal intensities for ACY1. The mean signal intensities for ACY1 autoantibody in HBV-related liver cirrhosis patients, CHB patients, and healthy controls were 3,791, 1,186 and 1,487 , respectively, with significant differences between HBV-related liver cirrhosis and CHB $(\mathrm{P}=0.001)$ or healthy controls $(\mathrm{P}=0.008)$ (Fig. 2B). The mean signal intensities for the other six autoantibodies with AUC values of $>0.7$ for discriminating HBV-related liver cirrhosis and $\mathrm{CHB}$ patients are shown in Fig. 3. 
A

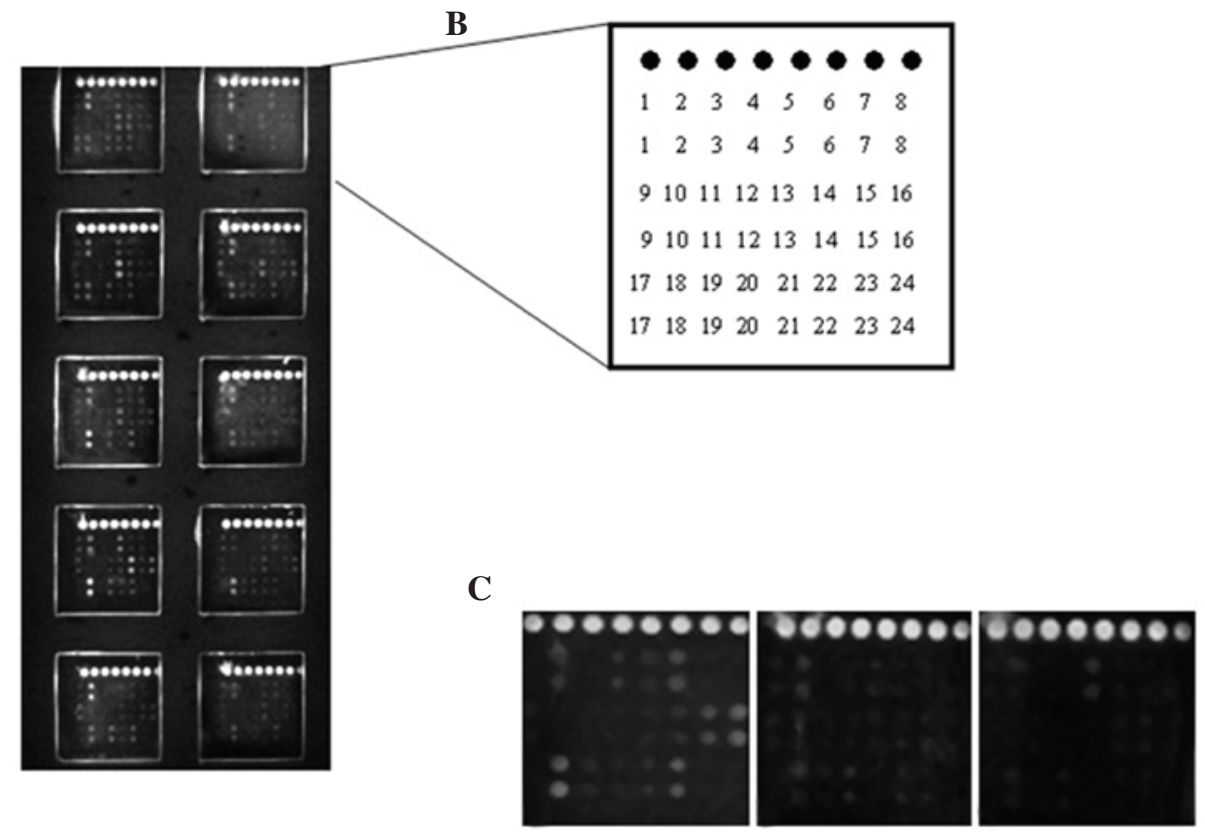

Figure 1. Schematic representation of the protein microarray for high-throughput clinical evaluation. (A) Scanned image of a representative antigen array. (B) Design of the protein microarray. The numbered spots correspond to the following antigens: •, IgG; 1, IGKC; 2, HSP60; 3, AIAT; 4, IMP-2; 5, FGB; 6, HSP A6; 7, ATPB; 8, BSA; 9, ALDH1A1; 10, PDIA1; 11, ENO1; 12, ANXA4; 13, CENPF; 14, SBP1; 15, ACY1; 16, hnRNP; 17, K2C1; 18, AIF; 19, CRT; 20, RGN; 21, PRDX3; 22, HINT1; 23, TUBB4B; 24, sample liquid. (C) Representative microarray detection of serum samples. Individual arrays were incubated with sera from HBV-related liver cirrhosis patients, CHB patient and healthy controls (left to right). IGKC, Ig $\kappa$ chain C region; HSP60, $60 \mathrm{kDa}$ heat shock protein; AIAT, $\alpha$-1-antitrypsin; IMP-2, insulin-like growth factor 2 mRNA-binding protein 2; FGB, fibrinogen $\beta$ chain; HSP A6, heat shock $70 \mathrm{kDa}$ protein 6; ATPB, ATP synthase subunit $\beta$; BSA, bovine serum aldumin; ALDH1A1, retinal dehydrogenase 1; PDIA1, protein disulfide-isomerase; ENO1, $\alpha$-enolase; ANXA4, annexin A4; CENPF, centromere protein F; SBP1, selenium-binding protein 1; ACY1, aminoacylase-1; hnRNP, heterogeneous nuclear ribonucleoprotein A2; K2C1, type II cytoskeletal 1; AIF, apoptosis-inducing factor; CRT, calreticulin; RGN, regucalcin; PRDX3, peroxiredoxin 3; HINT1, histidine triad nucleotide-binding protein 1; TUBB4B, tubulin $\beta-4 \mathrm{~B}$ chain.

$\mathbf{A}$

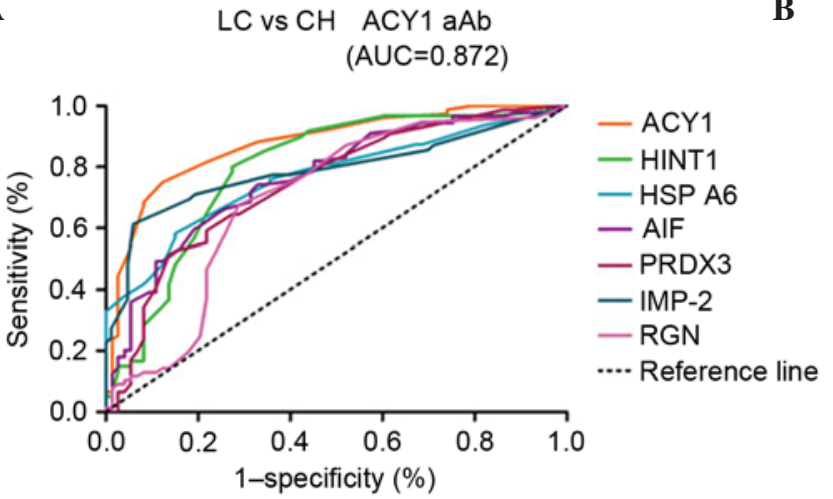

B

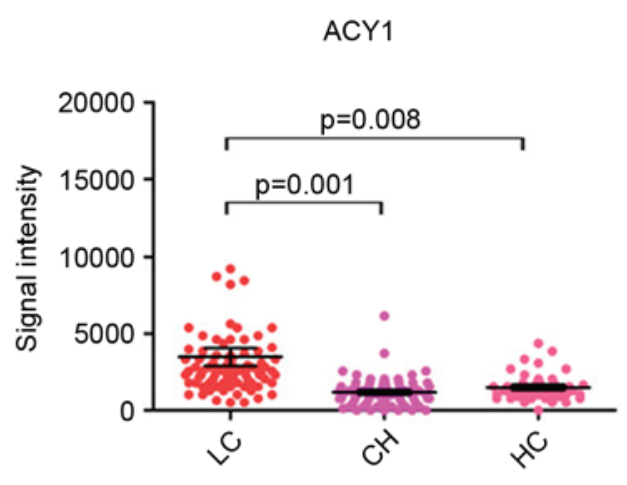

Figure 2. ROC curves of seven candidate autoantigens in discriminating between HBV-related liver cirrhosis and CHB with AUC values $>0.7$ and representative scatter diagram of the signal intensity of ACY1. (A) Receiver-operating characteristic curves of the seven autoantibodies for discriminating between HBV-related liver cirrhosis and CHB with AUC values $>0.7$. (B) Scatter diagram of the signal intensity of ACY1. The black horizontal lines indicate the mean values, and the error bars are standard errors. LC, liver cirrhosis; $\mathrm{CH}$, chronic hepatitis; HC, healthy controls; CHB, chronic hepatitis B; HBV, hepatitis B virus; AUC, area under the curve; ACY1, aminoacylase-1; HINT1, histidine triad nucleotide-binding protein 1; HSP A6, heat shock $70 \mathrm{kDa}$ protein 6; AIF, apoptosis-inducing factor; PRDX3, peroxiredoxin 3; IMP-2, insulin-like growth factor 2 mRNA-binding protein 2; RGN, regucalcin.

Prevalence of autoantibody positivity. Comparisons of the prevalence of autoantibody positivity against ACY1 and the other six protein antigens between HBV-related liver cirrhosis and $\mathrm{CHB}$ revealed significant differences in the numbers of patients with autoantibody positivity, with a prevalence of autoantibody positivity against $\mathrm{ACY} 1$ of $77.3 \%$ for $\mathrm{HBV}$-related liver cirrhosis and $15.0 \%$ for CHB $(\mathrm{P}<0.0001$; Table II $)$. Analyses of the associations with demographic parameters of HBV-related liver cirrhosis and CHB patients showed that the prevalence of the autoantibodies were not significantly correlated with gender and age (Table III).

\section{Discussion}

During the process of disease development, certain proteins may cause antigenicity through mutation, abnormal expression and abnormal localization, thereby triggering the immune system to produce autoantibodies $(10,11)$. Since the magnified 
Table II. Comparisons of autoantibody positivity among hepatitis B virus-related liver cirrhosis and CHB patients.

\begin{tabular}{lcccr}
\hline Autoantibody & Cut-off value & LC (\%) & CHB (\%) & P-value \\
\hline ACY1 & 1920 & 77.3 & 15.0 & $<0.0001$ \\
HINT1 & 1856 & 85.5 & 30.0 & $<0.0001$ \\
IMP-2 & 896 & 62.3 & 7.10 & $<0.0001$ \\
PRDX3 & 1728 & 54.5 & 10.0 & $<0.0001$ \\
HSP A6 & 1600 & 58.5 & 16.7 & $<0.0001$ \\
AIF & 5504 & 60.0 & 24.0 & $<0.0001$ \\
RGN & 640 & 86.1 & 46.7 & $<0.0001$
\end{tabular}

LC, liver cirrhosis; CHB, chronic hepatitis B; ACY1, aminoacylase-1; HINT1, histidine triad nucleotide-binding protein 1; IMP-2, insulin-like growth factor 2 mRNA-binding protein 2; PRDX3, peroxiredoxin 3; HSP A6, heat shock 70 kDa protein 6; AIF, apoptosis-inducing factor; RGN, regucalcin.

Table III. Correlation between autoantibody positivity and demographic parameters in hepatitis B virus-related liver cirrhosis and chronic hepatitis $\mathrm{B}$.

\begin{tabular}{|c|c|c|c|c|c|c|}
\hline \multirow[b]{3}{*}{ Autoantibody } & \multicolumn{6}{|c|}{ Prevalence of autoantibody positivity } \\
\hline & \multicolumn{2}{|c|}{ Gender } & \multirow[b]{2}{*}{ P-value } & \multicolumn{2}{|c|}{ Age (years) } & \multirow[b]{2}{*}{ P-value } \\
\hline & Male & Female & & $\geq 50$ & $<50$ & \\
\hline \multicolumn{7}{|l|}{ ACY1 } \\
\hline $\mathrm{LC}$ & $38 / 48(79.2 \%)$ & $13 / 18(72.2 \%)$ & 0.787 & $20 / 24(83.3 \%)$ & $31 / 42(73.8 \%)$ & 0.374 \\
\hline $\mathrm{CH}$ & $5 / 38(13.2 \%)$ & $4 / 22(18.2 \%)$ & 0.881 & $7 / 41(17.1 \%)$ & $2 / 19(10.5 \%)$ & 0.786 \\
\hline \multicolumn{7}{|l|}{ HINT1 } \\
\hline $\mathrm{LC}$ & $33 / 37(89.2 \%)$ & $14 / 18(77.8 \%)$ & 0472 & $16 / 20(80.0 \%)$ & $31 / 35(88.6 \%)$ & 0.638 \\
\hline $\mathrm{CH}$ & $11 / 38(28.9 \%)$ & $7 / 22(31.8 \%)$ & 0.815 & $14 / 45(31.1 \%)$ & $4 / 15(26.7 \%)$ & 1.000 \\
\hline \multicolumn{7}{|l|}{ IMP-2 } \\
\hline $\mathrm{LC}$ & $26 / 44(59.1 \%)$ & $12 / 17(70.6 \%)$ & 0.406 & $17 / 25(68.0 \%)$ & $21 / 36(58.3 \%)$ & 0.444 \\
\hline $\mathrm{CH}$ & $4 / 47(8.5 \%)$ & $1 / 23(4.3 \%)$ & 0.525 & $4 / 55(7.3 \%)$ & $1 / 15(6.7 \%)$ & 1.000 \\
\hline \multicolumn{7}{|l|}{ PRDX3 } \\
\hline $\mathrm{LC}$ & $26 / 46(56.5 \%)$ & $10 / 20(50.0 \%)$ & 0.625 & $10 / 25(40.0 \%)$ & $26 / 41(63.4 \%)$ & 0.064 \\
\hline $\mathrm{CH}$ & $6 / 40(15.0 \%)$ & $0 / 20(0.00 \%)$ & 0.171 & $3 / 44(6.80 \%)$ & $3 / 16(18.8 \%)$ & 0.381 \\
\hline \multicolumn{7}{|l|}{ HSP A6 } \\
\hline $\mathrm{LC}$ & $30 / 47(63.8 \%)$ & $8 / 18(44.4 \%)$ & 0.156 & $14 / 26(53.8 \%)$ & $24 / 39(61.5 \%)$ & 0.538 \\
\hline $\mathrm{CH}$ & $8 / 39(20.5 \%)$ & $2 / 21(9.50 \%)$ & 0.468 & $7 / 47(14.9 \%)$ & $3 / 13(23.1 \%)$ & 0.779 \\
\hline \multicolumn{7}{|l|}{ AIF } \\
\hline $\mathrm{LC}$ & $35 / 57(61.4 \%)$ & $13 / 23(56.5 \%)$ & 0.687 & $22 / 33(66.7 \%)$ & $26 / 47(55.3 \%)$ & 0.308 \\
\hline $\mathrm{CH}$ & $8 / 38(21.1 \%)$ & $4 / 22(18.2 \%)$ & 1.000 & $10 / 44(22.7 \%)$ & $2 / 16(12.5 \%)$ & 0.609 \\
\hline \multicolumn{7}{|l|}{ RGN } \\
\hline $\mathrm{LC}$ & $43 / 51(84.3 \%)$ & $19 / 21(90.5 \%)$ & 0.755 & $23 / 29(79.3 \%)$ & $39 / 43(90.7 \%)$ & 0.306 \\
\hline $\mathrm{CH}$ & $18 / 38(47.4 \%)$ & $10 / 22(45.5 \%)$ & 0.886 & $20 / 36(55.6 \%)$ & $8 / 24(33.3 \%)$ & 0.091 \\
\hline
\end{tabular}

ACY1, aminoacylase-1; HINT1, histidine triad nucleotide-binding protein 1; IMP-2, insulin-like growth factor 2 mRNA-bindin protein 2; HSP A6, heat shock $70 \mathrm{kDa}$ protein 6; PRDX3, peroxiredoxin 3; AIF, apoptosis-inducing factor; RGN, regucalcin; LC, liver cirrhosis; $\mathrm{CH}$, chronic hepatitis.

signals of autoantibodies can be easier to detect than autoantigens themselves $(3,7)$, the autoantibody based serum biomarkers may exhibit higher sensitivity than protein antigens, and may be promising biomarkers for early diagnosis of diseases.
Current studies on serum autoantibodies are predominantly focused on autoimmune diseases, such as systemic lupus erythematosus, rheumatoid arthritis and autoimmune liver diseases. In liver diseases, screening of autoantibodies is mainly used 

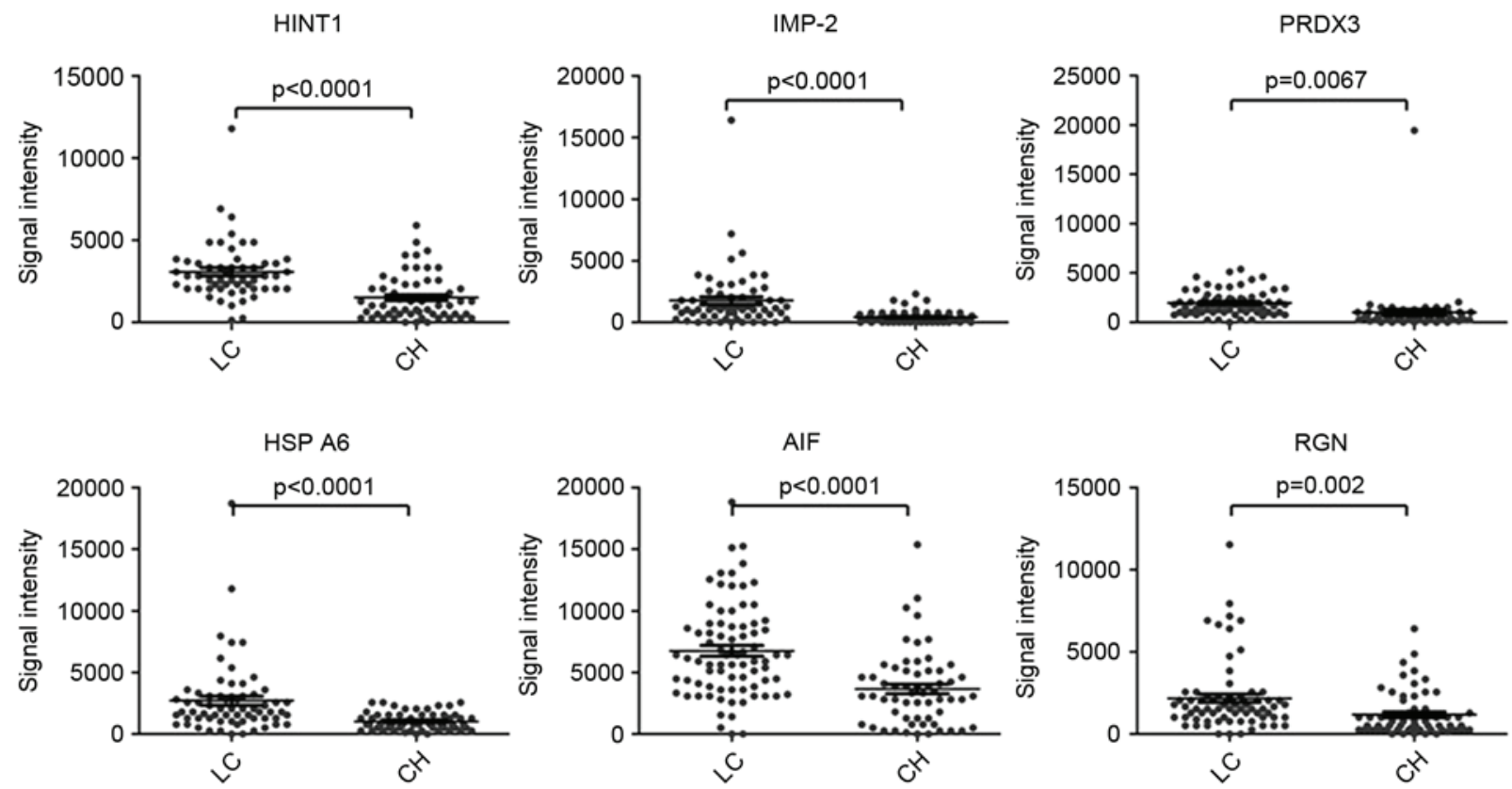

Figure 3. Representative scatter diagrams of the signal intensity of HINT1, IMP-2, PRDX3, HSP A6, AIF and RGN for detection of LC and CH. The black horizontal lines indicate the mean values, and the error bars are standard errors. LC, liver cirrhosis; CH, chronic hepatitis; HINT1, histidine triadnucleotide-binding protein 1; IMP-2, insulin-like growth factor 2 mRNA-binding protein 2; PRDX3, peroxiredoxin 3; HSP A6, heat shock 70 kDa protein 6; AIF, apoptosis induced factor; RGN, regucalcin.

to aid in the diagnosis of autoimmune liver diseases, such as autoimmune hepatitis and primary biliary cirrhosis. A number of autoimmune liver disease-related autoantibodies, such as antinuclear antibodies and antimitochondrial antibodies have been reported (12). However, the underlying mechanism of the generation of these autoantibodies in autoimmune diseases remains poorly understood. Autoantibodies have also been observed in non-autoimmune diseases, such as cancer (3-5). In the present study, several autoantibodies were identified with different levels in the serum of CHB and HBV-related liver cirrhosis patients without autoimmune diseases.

Protein microarray technology was applied in the present study for high throughput clinical evaluation of the candidate protein antigens. The level of CENPF autoantibody was detected by western blot analysis in out previous study and the results were consistent with that of the protein microarray (8), suggesting the robust results obtained by the microarray assays in our study. The protein antigens with significant difference of signal intensity between HBV-related liver cirrhosis and CHB screened by the present study were classified as follows: Proteins involved in construction of the cytoskeleton and promotion of cell proliferation and activation, such as CENPF, ANXA4, TUBB4B, K2C1 and FGB; proteins related to metabolism and transport, such as ALDH1A1, ENO1, ATPB, ACY1, PRDX3 and RGN; proteins involved in the immune system, such as IGKC and HINT1, and stress-related proteins, such as HSP60, HSPA6 and PDIA1. Among these candidate autoantigens, ENO1, ACY1 and HINT1 were previously reported to be associated with fibrosis $(6,13,14)$.

As the most valuable candidate biomarker for liver cirrhosis screened in the present study, ACY1 is associated with protein metabolism, participates in protein degradation, and was found to be associated with colon cancer, small cell lung cancer and liver cancer (15-17). In a previous study, mice with renal fibrosis were treated with the antifibrotic drug mycophenolate mofetil, and evaluated for the protein abundance in kidney sections (13). The results showed the level of ACY1 was elevated, while tubulointerstitial fibrosis was inhibited by mycophenolate mofetil. These observations indicated that ACY1 may be involved in the process of fibrosis, but its specific function remained unclear. In the present study, to the best of our knowledge, it was demonstrated for the first time that the level of ACY1 autoantibody was higher in patients with HBV-related liver cirrhosis compared with patients with CHB. However, further studies are required to clarify the mechanism underlying the generation of the ACY1 autoantibody.

HINT1 is a novel tumor inhibiter identified in recent years $(18,19)$. It is implicated in the pathological progression of a number of human diseases, including cancer and schizophrenia (20). Wu et al (14) found that rhHint1 was capable of attenuating $\mathrm{CCl}_{4}$-induced liver fibrosis in rats by simultaneously targeting multiple pathogenic pathways, and may have potential for development as a novel treatment for liver fibrosis. The present study demonstrated that the level of HINT1 autoantibody in patients with HBV-related liver cirrhosis was higher than that in patients with CHB. However, further studies on the mechanism underlying the generation of HINT1 autoantibody are required.

Consistent with the study by Peng et al (6) in which ENO1 was identified as a potential marker for liver fibrosis, ENO1 autoantibody was present at a significantly higher level in patients with HBV-related liver cirrhosis compared with patients with CHB in the present study. Enolases are a family of cytoplasmic proteins involved in glycolytic metabolism and energy regulation in prokaryotes and eukaryotes. ENO1 is located in the nucleus and aids in the regulation of cell growth and differentiation by downregulating the activity of the proto-oncogene c-myc (21). ENO1 autoantibody has been found in autoimmune diseases, such as systemic lupus erythematosus, rheumatoid arthritis and 
Crohn's disease, and also appeared in autoimmune hepatitis with a low titer (22-24). Peng et al (6) found that the frequency of ENO1 autoantibodies in sera from patients at the precirrhotic stage of liver fibrosis $(21.6 \%, 27 / 125)$ was significantly higher than that in sera from patients with cirrhosis $(9.1 \%, 5 / 55)$ and liver cancer $(14.3 \%, 12 / 84)$, as well as that in sera of healthy individuals $(4.1 \%, 3 / 74)$.In the present study, it was demonstrated that the level of the ENO1 autoantibody was higher in patients with HBV-related cirrhosis compared with patients with $\mathrm{CHB}$ $(\mathrm{P}<0.05)$. Therefore, ENO1 may be an autoantigen that elicits autoimmune responses in liver fibrosis and it may be a potential prognostic factor for liver fibrosis diagnosis.

Liver fibrosis is an important stage of the progression from chronic hepatitis to cirrhosis and hepatocellular carcinoma. Increasing evidence suggests that liver fibrosis is reversible, even for advanced fibrosis (25). Early detection and accurate predication of the degree of fibrosis are crucial for the prevention of liver cirrhosis and carcinoma. A liver biopsy has traditionally been used as the golden standard for liver fibrosis, but the process is unpleasant for patients and may have severe side effects, such as local hemorrhage and pain caused by liver puncture, and pneumothorax in a small number of patients $(26,27)$. Since fibrosis not only occurs in the liver, but also in the kidney, lung, and skin, poor specificity is a common problem of frequently-used serum biomarkers, such as hyaluronic acid, laminin, metal matrix proteinase, tissue inhibitor of matrix metalloprotease-1 and cytokines (28). Although a number of studies have shown that transient elastography performs well in the assessment of significant and advanced fibrosis $(29,30)$, its reproducibility was reported to be lower in patients with steatosis, increased body mass index, lower degrees of hepatic fibrosis and severe ascites (31). Therefore, a noninvasive method with high diagnostic value and specificity is strongly required.

Few studies have addressed the value of an autoantibody-based serum biomarker in the staging of liver fibrosis. The data showed that autoantibodies against ACY1, HINT1, IMP-2, PRDX3, HSPA6, AIF and RGN may be useful in the discrimination of HBV-related liver cirrhosis and CHB. Since liver fibrosis is the intermediate stage of the progress from chronic hepatitis to liver cirrhosis, the screened candidate autoantibody biomarkers may have further potential value in the diagnosis of liver fibrosis of different stages. Another study may be conducted in the future to evaluate the efficacy of these autoantibodies in liver fibrosis and analyze the underlying mechanism of the appearance of these autoantibodies.

One limitation of the present study is that all of the cases of liver cirrhosis and hepatitis were caused by HBV infection. Although hepatitis $\mathrm{C}$ virus infection and alcohol abuse are also important causes of liver cirrhosis, the effects of these factors were not investigated in this study. However, future investigation into the contributions of these factors is required. In addition, the number of participants enrolled in the present study was insufficient, thus a large-scale study is required. In summary, with the elevated level in the disease progression of CHB, ACY1 autoantibody maybe a valuable serum biomarker for discriminating $\mathrm{HBV}$-associated liver cirrhosis from $\mathrm{CHB}$, providing a convenient method for the screening and early diagnosis of patients with liver cirrhosis, and the basis for further research on autoantibody-based biomarkers for the staging of liver fibrosis.

\section{Acknowledgements}

The authors would like to thank Dr Jidong Jia, Dr Hong You and Dr Hong Ma (Liver Research Center, Beijing Friendship Hospital, Capital Medical University) for supplying clinical materials and helpful suggestion. This study was supported by a grant from the National Natural Science Foundation of China (grant no. 81071973), a grant from the Scientific Research Foundation for Returned Overseas Chinese Scholars, Bureau of Human Resources and Social Security of Beijing, China (Key project, 2010, grant no. 20110323), and a grant from the program for National Science and Technology Major Project (grant no. 2013ZX10002004).

\section{References}

1. European Association For The Study Of The Liver: EASL Clinical Practice Guidelines: Management of chronic hepatitis B. J Hepatol 50: 227-242, 2009.

2. Bini EJ and Perumalswami PV: Hepatitis B virus infection among American patients with chronic hepatitis $\mathrm{C}$ virus infection: Prevalence, racial/ethnic differences, and viral interactions. Hepatology 51: 759-766, 2010.

3. Tan HT, Low J, Lim SG and Chung MC: Serum autoantibodies as biomarkers for early cancer detection. FEBS J 276: 6880-6904, 2009.

4. Xu YW, Peng YH, Chen B, Wu ZY, Wu JY, Shen JH, Zheng CP, Wang SH, Guo HP, Li EM and Xu LY: Autoantibodies as potential biomarkers for the early detection of esophageal squamous cell carcinoma. Am J Gastroenterol 109: 36-45, 2014.

5. Werner S, Chen H, Tao S and Brenner H: Systematic review: Serum autoantibodies in the early detection of gastric cancer. Int J Cancer 136: 2243-2252, 2015.

6. Peng B, Huang X, Nakayasu ES, Petersen JR, Qiu S, Almeida IC and Zhang JY: Using immunoproteomics to identify alpha-enolase as an autoantigen in liver fibrosis. J Proteome Res 12: 1789-1796, 2013.

7. Lacombe J, Mangé A and Solassol J: Use of autoantibodies to detect the onset of breast cancer. J Immunol Re 2014: 574981, 2014.

8. Hong Y, Long J, Li H, Chen S, Liu Q, Zhang B, He X, Wang Y, $\mathrm{Li} \mathrm{H,} \mathrm{Li} \mathrm{Y,} \mathrm{et} \mathrm{al:} \mathrm{An} \mathrm{analysis} \mathrm{of} \mathrm{immunoreactive} \mathrm{signatures} \mathrm{in} \mathrm{early}$ stage hepatocellular carcinoma. EBioMedicine 2: 438-446, 2015.

9. Lok AS and McMahon BJ: Chronic hepatitis B: Update 2009. Hepatology 50: 661-662, 2009.

10. Casiano CA, Mediavilla-Varela M and Tan EM: Tumor-associated antigen arrays for the serological diagnosis of cancer. Mol Cell Proteomics 5: 1745-1759, 2006.

11. Caron M, Choquet-Kastylevsky G and Joubert-Caron R: Cancer immunomics using autoantibody signatures for biomarker discovery. Mol Cell Proteomics 6: 1115-1122, 2007.

12. Nakamura M: Clinical significance of autoantibodies in primary biliary cirrhosis. Semin Liver Dis 34: 334-340, 2014.

13. Petrova DT, Brehmer F, Schultze FC, Asif AR, Gross O, Oellerich M and Brandhorst G: Differential kidney proteome profiling in a murine model of renal fibrosis under treatment with mycophenolate mofetil. Pathobiology 78: 162-170, 2011.

14. Wu F, Huang S, Zhu N, Liu W, Zhang Y and He Y: Recombinant human histidine triad nucleotide-binding protein 1 attenuates liver fibrosis induced by carbon tetrachloride in rats. Mol Med Rep 8: 1023-1038, 2013.

15. Miller YE, Minna JD and Gazdar AF: Lack of expression of aminoacylase-1 in small cell lung cancer. Evidence for inactivation of genes encoded by chromosome 3p. J Clin Invest 83 : 2120-2124, 1989

16. Shi H, Hayes MT, Kirana C, Miller RJ, Keating JP and Stubbs RS: Overexpression of aminoacylase 1 is associated with colorectal cancer progression. Hum Pathol 44: 1089-1097, 2013.

17. Wei X, Li J, Xie H, Ling Q, Wang J, Lu D, Zhou L, Xu X and Zheng S: Proteomics-based identification of the tumor suppressor role of aminoacylase 1 in hepatocellular carcinoma. Cancer Lett 351: 117-125, 2014.

18. Li H, Zhang Y, Su T, Santella RM and Weinstein IB: Hint1 is a haplo-insufficient tumor suppressor in mice. Oncogene 25: 713-721, 2006. 
19. Weiske $\mathbf{J}$ and Huber O: The histidine triad protein Hint1 triggers apoptosis independent of its enzymatic activity. J Biol Chem 281: 27356-27366, 2006.

20. Dang YH, Liu ZW, Chen F, Guo K and Wang JB: Histidine triad nucleotide-binding protein 1 and human diseases. Zhongguo $\mathrm{Yi}$ Xue Ke Xue Yuan Xue Bao 36: 454-460, 2014 (In Chinese).

21. Terrier B, Degand N, Guilpain P, Servettaz A, Guillevin L and Mouthon L: Alpha-enolase: A target of antibodies in infectious and autoimmune diseases. Autoimmun Rev 6: 176-182, 2007.

22. Kinloch A, Tatzer V, Wait R, Peston D, Lundberg K, Donatien P, Moyes D, Taylor PC and Venables PJ: Identification of citrullinated alpha-enolase as a candidate autoantigen in rheumatoid arthritis. Arthritis Res Ther 7: R1421-R1429, 2005.

23. Roozendaal C, Zhao MH, Horst G, Lockwood CM, kleibeuker JH, Limburg PC, Nelis GF and Kallenberg CG: Catalase and alpha-enolase: Two novel granulocyte autoantigens in inflammatory bowel disease (IBD). Clin Exp Immunol 112: 10-16, 1998.

24. Pratesi F, Moscato S, Sabbatini A, Chimenti D, Bombardieri S and Migliorini P: Autoantibodies specific for alpha-enolase in systemic autoimmune disorders. J Rheumatol 27: 109-115, 2000.

25. Friedman SL: Liver fibrosis-from bench to bedside. J Hepatol 38 (Suppl 1): S38-S53, 2003.
26. Siddique I, El-Naga HA, Madda JP, Memon A and Hasan F: Sampling variability on percutaneous liver biopsy in patients with chronic hepatitis C virus infection. Scand J Gastroenterol 38: 427-432, 2003.

27. Rockey DC, Caldwell SH, Goodman ZD, Nelson RC and Smith AD; American Association for the Study of Liver Diseases: Liver biopsy. Hepatology 49: 1017-1044, 2009.

28. Liu T, Wang X, Karsdal MA, Leeming DJ and Genovese F Molecular serum markers of liver fibrosis. Biomark Insights 7: 105-117, 2012.

29. Chon YE, Choi EH, Song KJ, Park JY, Kim do Y, Han KH, Chon CY, Ahn SH and Kim SU: Performance of transient elastography for the staging of liver fibrosis in patients with chronic hepatitis B: A meta-analysis. PLoS One 7: e44930, 2012.

30. Verveer C, Zondervan PE, ten Kate FJ, Hansen BE, Janssen HL and de Knegt RJ: Evaluation of transient elastography for fibrosis assessment compared with large biopsies in chronic hepatitis B and C. Liver Int 32: 622-628, 2012.

31. Fraquelli M, Rigamonti C, Casazza G, Conte D, Donato MF, Ronchi G and Colombo M: Reproducibility of transient elastography in the evaluation of liver fibrosis in patients with chronic liver disease. Gut 56: 968-973, 2007. 